\title{
REAL-LINK PERFORMANCE OF A SS-MC-MA HIGH FREQUENCY RADIO MODEM
}

\author{
Héctor Santana-Sosa ${ }^{1}$, Ivana Raos ${ }^{2}$, Santiago Zazo-Bello ${ }^{2}$, Iván A. Pérez- \\ Álvarez ${ }^{1}$ and Javier López-Pérez ${ }^{1}$ \\ ${ }^{1}$ Dpto. Señales y Comunicaciones \\ Universidad de Las Palmas de Gran Canaria \\ Las Palmas de Gran Canaria, Spain \\ $\{$ hector, ivan, javivi\}@gic.dsc.ulpgc.es \\ ${ }^{2}$ Centro de Domótica Integral \\ Universidad Politécnica de Madrid \\ Madrid, Spain \\ \{ivana, santiago\}@gaps.ssr.upm.es
}

\begin{abstract}
The HF band data communication link has been traditionally desired by many of the large range transmission systems although it is associated to unfavorable performances as low transmission rate, large delay and low confidence in terms of link establishment and maintenance. Although transmission rates may be high enough to transmit digital voice, delay, usually over several second, has been the main handicap to let the systems provide interactive digital voice links. Indeed, there is no unclassified equipment with this capability. The main achievement of this proposal is that we are able to guarantee digital voice transmission with low latency, around $135 \mathrm{msec}$ (modem+codec), providing a full interactive digital voice link. Performances of two new $2460 \mathrm{bps}$. HF modems are presented versus the 39 -tone 2400 bps MIL-STD-188-110A modem, working over an ITU-R moderate channel. Futhermore, these results are corroborated by real tests carried out in a $1800 \mathrm{Km}$. link.
\end{abstract}

\section{Introduction}

\subsection{The HF Channel}

In the HF band, long distance communications are feasible thanks to the use of the ionosphere as passive reflector. However, the atmospheric nature of this reflector makes the systems to face a very hard commu- 


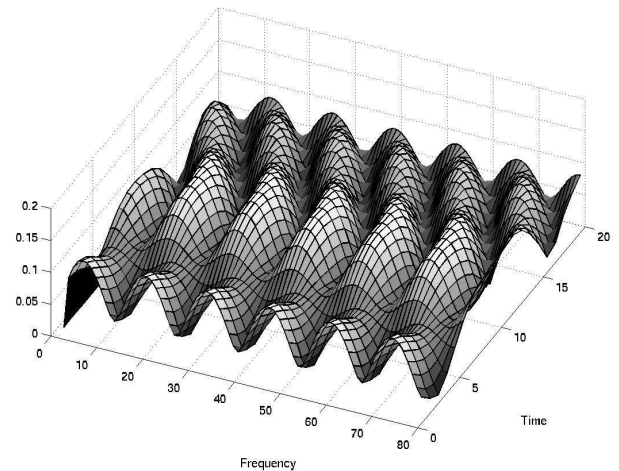

Figure 1. Time-Frequency representation of the HF channel

nication environment. Multipath effects are always present and have to be considered in depth, beside the very fast time-varying characteristics of the channel. The main parameters used to measure channel behavior are frequency coherence $\left(\Delta f_{c}\right)$ and time coherence $\left(\Delta t_{c}\right)[12]$. The first one $\left(\Delta f_{c}\right)$ gives information about how narrow must one modulated carrier be in order to consider the channel flat. Typical values in HF channel are close to $1 \mathrm{KHz}$ or less. The second parameter $\left(\Delta t_{c}\right)$ sets the time separation between two pulses with different attenuations. Its inverse is known as doppler spread $\left(f_{d}\right)$ and in HF it is usually about 0.1 to $2 \mathrm{~Hz}$. This multipath environment makes efficiencies over $0.5 \mathrm{bit} / \mathrm{Hz}$ very difficult to achieve. It is common to find channel characteristics as shown in Figure 1, where several deep nulls spread over the narrow transmission band and move arbitrarily over it. Usually, the bandwidth of $\mathrm{HF}$ transmissions are less than $3 \mathrm{KHz}$ and the best performances in data communications are obtained usually by the combination of powerful codes and very long interleavers, which introduce an important delay in the communication and make interactive digital voice link impossible.

\subsection{State of the Art}

Single carrier schemes have been typically used for facing the hard conditions of the short-wave radio communications. Indeed, most of the systems designed for data transmission in the HF band use single carrier techniques beside powerful coding [6]. Optimum coding performance needs the use of long interleaving matrices in order to cope with burst errors introduced by the channel. Interactive digital voice communications are not feasible with this kind of systems due to this unacceptable delay. Most of present data modem applications are based 
on the standard MIL-STD-188-110A [9] as a military data transmission system, or in the standard STANAG 4285 [15]. The modem described by the standard MIL-STD-188-110A [9] specifies data rates range between 75 and 2400 bps. Transmission process uses an 8-states convolutional encoder with 8PSK modulation scheme with variable interleavers. This standard also describes in two appendixes, two multicarrier schemes: one of them is non-orthogonal 16 tones DPSK where channel estimation is not required, but also spectral efficiency is reduced. The other operating mode is an orthogonal (OFDM) 39-tones including a $(14,10)$ Reed-Solomon encoder with a frequency diversity degree selected depending on the channel state. This modem has to be considered as one of the first HF-band military systems published in the open literature and thus, it is one reference for this kind of systems although it does not provide support for an interactive digital voice link. Indeed, nowadays, there are no unclassified systems to support this kind of links.

\subsection{Multicarrier Modulations}

One of the main problems to consider when a HF modem is being designed is related to the long impulse response length of the channel. The first strategy to deal with this problem is to reduce transmission rate reducing multipath distortion to a small symbol fraction. Required data transmission rate might be obtained by means of Multi-Carrier techniques, and in particular with Orthogonal Frequency Division Multiplex (OFDM), thus giving maximum spectral efficiency. The performances of OFDM schemes in HF channels have been deeply analyzed by C.Cook [4], also published by E.E. Johnson [6], considering a large set of parameters as interleaving length, robustness against impulsive noise, behavior in front of co-channel interference, relationship between average and peak power, equipment specifications, synchronization issues, and spatial diversity techniques applicability. In these studies, OFDM based modems have demonstrated to be more efficient than single carrier systems when long interleaving and powerful coding are avoided.

\section{Data Modem Design}

Two schequematic block diagrams of the developed modem are presented in Figure 2 and Figure 3. They represent the transmitter and the receiver respectively. The design of the new modem has been focused on three main topics: the use of continuos channel sounding distributed in the frequency-time grid, the use of MC-CDMA techniques to avoid deep-nulls effects, and completely avoid coding and interleaving to provide interactive digital voice links. 


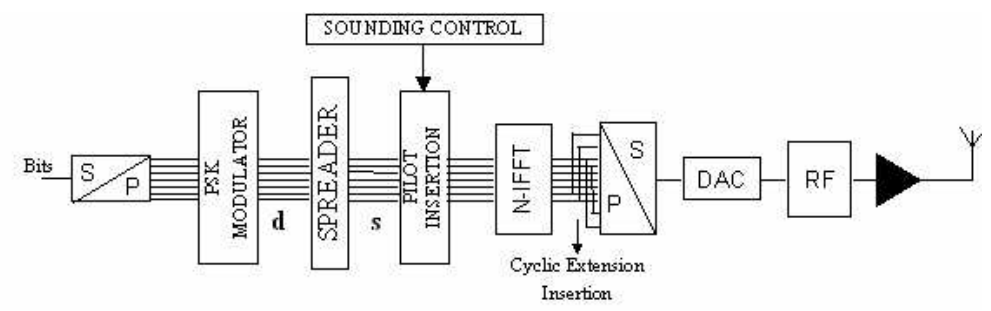

Figure 2. Block diagram of the transmiter

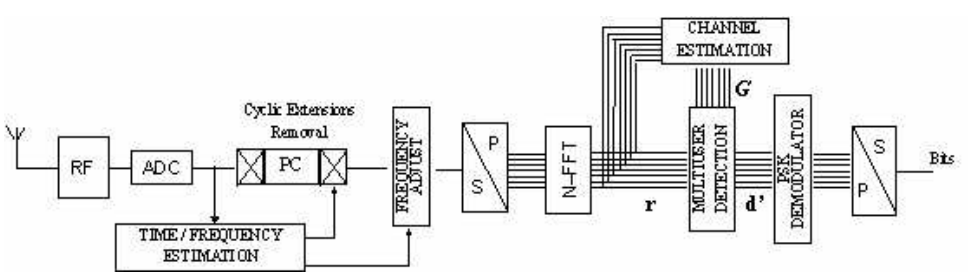

Figure 3. Block diagram of the receiver

\subsection{SS-MC-MA Techniques}

SS-MC-MA techniques provide a robust mechanism to avoid the effects of the deep-nulls introduced by the channel (Figure 1) without the need of powerful coding and long interleaving. Work showed in [14] and [11] firstly introduced these techniques in this kind of systems and demonstrated, in a simple simulated environment, that SS-MC-MA is a valid technique to provide interactive digital voice communications over ionospheric links. Using SS-MC-MA, the serial symbols are transmitted over all frequencies so the information affected by deep-nulls can be restored with the unaffected carriers using frequency diversity mechanism.

Taking [14] and [11] as a starting point, this paper presents a step forward in the field of SS-MC-MA techniques applied to short-wave radio modems. As it was seen in the block diagrams, coding has been completely removed from the previous versions as its benefits with no interleaving have been overcomed by MC-CDMA techniques. These techniques are common in researches related to new mobile communications systems (i.e. [7]) but have not been exploited in this kind of systems. The lack of coding and interleaving is not a problem as the new advanced reception patterns have increased performance thanks to the correct use of frequency diversity. Different detection strategies are used such as Global Minimum Mean Square Error (GMMSE) and Interference Cancellation techniques [2], all of them adapted in order to fit into new transmission schemes. Two new modems have been developed. One of them uses 


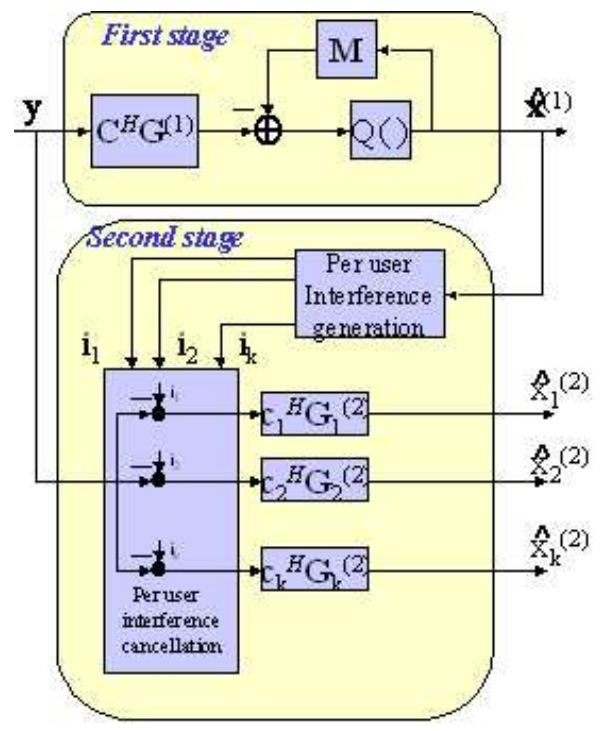

Figure 4. Interference Cancellation Process

GMMSE with a two-stages Interference Cancellation scheme. The second one performs a Parallel Interference Cancellation (PIC) following the hints given in [14] and [11] with Equal Gain Combining (EGC). The combination of GMMSE with standard PIC structures is straightforward as can be observed in the Figure 4; where $Q()$ is the standard symbol detector by minimum distance criteria and $\mathbf{M}$ represents the interference regeneration process:

$$
\mathbf{M}=\left(\mathbf{C}^{H} \mathbf{G H C}-\operatorname{diag}\left(\mathbf{C}^{H} \mathbf{G H C}\right)\right)
$$

where $\operatorname{diag}()$ operator means a diagonal matrix whose inputs are the corresponding elements of the processed matrix, $\mathbf{G}$ is a matrix calculated following GMMSE [2] criteria, $\mathbf{C}$ represents the spreading matrix where $\mathbf{c}_{k}$ is its k-column and $\mathbf{i}_{k}$ is the interference of the whole users over the $\mathrm{k}$-user.

\section{Results Analysis}

Simulated results are shown in the Figure 5, where MIL-STD 39 tones modem ( [6], [4] and [9]) efficiency are presented beside the two new modems performances. These results were obtained with a Moderate Channel Watterson HF Channel (2 rays, time spread 1 msec, Doppler spread $0.5 \mathrm{~Hz}$ ). The two new modems operate at $2460 \mathrm{bps}$ and the 39-tone MIL-STD-188-110A operates at 2400 bps. It can be seen that 


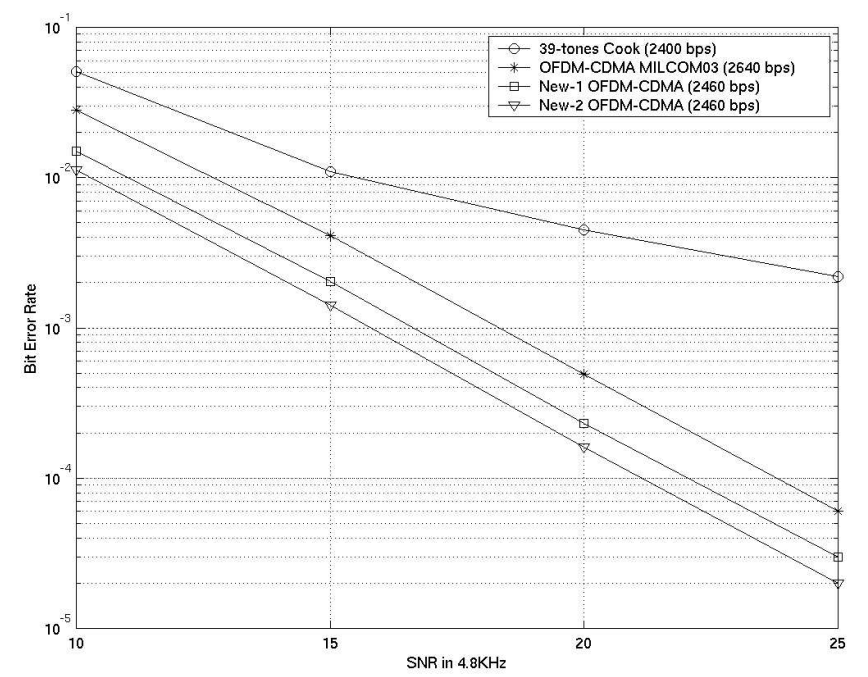

Figure 5. Performance of the two new modems vs. 39-tone MIL-STD-188-110A and $[14]$

the two new modems overcomes the MIL-STD performace. It is very important to recall that these results are obtained without any kind of interleaving matrix, so the data delay is kept extremely low. This delay does not goes over 135 msec Considering that the 2400 bps MELP vocoder [8] will operate satisfactorily with error probabilities below $10^{-2}$, we can guarantee modem operability for SNR in $4.8 \mathrm{KHz}$ above $10 \mathrm{~dB}$.

It is also presented in the Figure 6 the performance of the two new modems in a five hours real link. This link was established between Canary Islands and Madrid in the $18 \mathrm{MHz}$ band. It can be seen the parallelism between the simulated results and the real link performances. The main difference between the simulated and real link results is a SNR offset. This difference have its explanation in the fact that no real and variable channel will behave as a simulated CCIR channel. Deeper studies are considered in order to obtain actual channel conditions. Nevertheless, these results suppose a step forward in the way of demonstrating the system robustness.

\section{Conclusions and Future Research Lines}

Present work has shown the real feasibility of interactive digital voice transmission over the HF channel. This accomplishes a significant achievement in comparison with conventional analog transmission with a sig- 


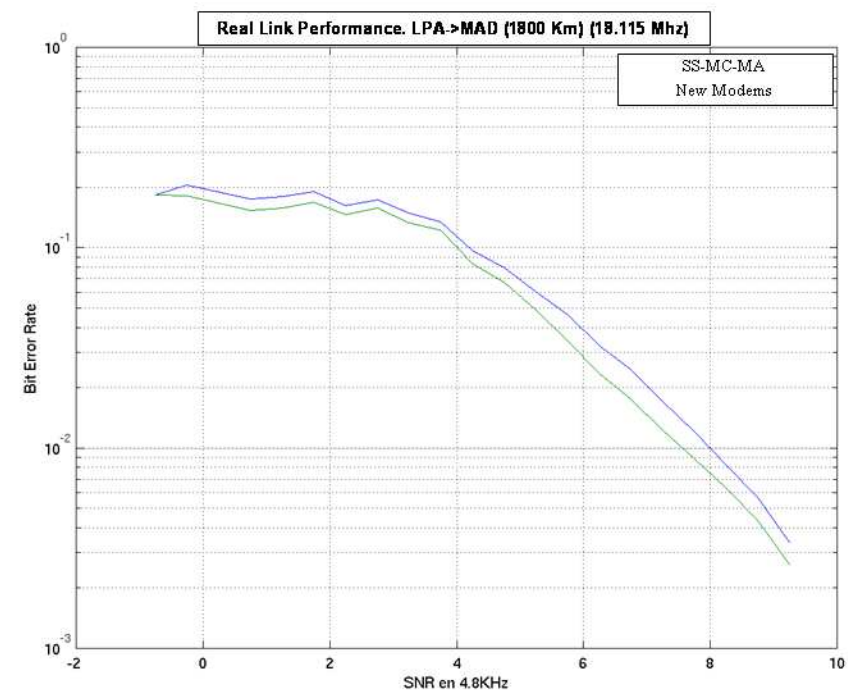

Figure 6. Performance of the two new modems in a 5 hours real link

nificant subjective improvement in terms of quality and intelligibility. The key issues are the following: the use of OFDM modulation and the use of MC-CDMA techniques inspired in mobile applications, increasing system robustness in front of deep spectral nulls and homogenizing performance over different subcarriers. Furthermore, once the transmission scheme has demonstrated to be efficient, complementary analysis, relaxing the delay constraint for data transmission [13], is a promising path to achieve new goals. This operation mode will increase data rate, by the increase of the constellation size and the use of combined interleaving and channel coding strategies.

\section{Acknowledgments}

The work presented in this contribution was supported by the Spanish National Project TEC2004-06915-C03-01/02 and AENA (Spanish Airports and Air Navigation) projects 00/49 and 00/50. It is also partially supported by the Spanish National Project TIC2003-09061-C03-01

\section{References}

[1] Khaled Fazel and Stefan Kaiser, Multi-Carrier and Spread Spectrum Systems. John Wiley and Sons, 2003.

[2] J.-Y. Daudais, J.-F. Hélard, J. Citerne. An Improved Linear MMSE Detection Tecnique for MultiCarrier CDMA Systems: Comparison and Combination with 
H. Santana-Sosa, I. Raos, S. Zazo-Bello, I. Pérez-Álvarez, J. López-Pérez

Interference Cancellation Schemes. European Transaction on Telecommunications. ETT Vol. 11, No.6, pages 547-554, November/December 2000.

[3] CCIR, Rc. 520-1. On the Use of ionospheric channel simulators in decametric waves. International Telecommunications Union (ITU), 1982.

[4] Cook, S.C. Advanced in high speed HF Radio modem design. Proceedings of Nordic Shortwave Conference (HF95), Faro (Sweden) 1995.

[5] Fernández-Getino, et al. Novel pilot patterns for channel estimation in OFDM mobile systems over frequency selective fading channels. Proceedings IEEE International Symposium on Personal Indoor, Mobile and Radio Communications, PIMRC?99, Osaka, Japan , 1999

[6] E.E. Johnson, et al. Advanced High Frequency Radio Communications. Artech House 1997.

[7] Matrice project web site: www.ist-matrice.org

[8] Draft to Federal Information Processing Standards Publication. Analog to Digital Conversion of Voice by 2400 bit/second Mixed Excitation Linear Prediction (MELP) , May, 1998.

[9] Department of the Army, Information Systems Engineering Command, MILSTD-188-110A: Interoperability and Performance Standards for Data Modems. Philadelphia, PA. Naval Publications and Forms Center, Attn. NPODS, Sep 1991.

[10] J. Pennington. Techniques for medium speed data transmission over HF channels. IEE Proceedings Vol.136, Part I, No. 1, February 1989, pp. 11-19.

[11] I. Pérez-Álvarez, I. Raos, S. Zazo, E. Mendieta-Otero, H. Santana-Sosa, J.M. Páez-Borrallo.Interactive Digital Voice Over HF. Ninth International Conference on HF Radio Systems and Techniques. Bath, U.K. June 2003.

[12] Proakis, J.G. Digital Communications. McGraw-Hill, Inc., pages 702-782, 2nd edition, 1989.

[13] I. Raos, A. Del Cacho, I. Pérez-Álvarez, S. Zazo, E. Mendieta-Otero, H. Santana-Sosa, J.M. Páez-Borrallo. Advanced OFDM-CDMA HF Modem with SelfInterference Cancellation. Milcom?03. Boston (USA). October 2003.

[14] I. Raos, A. Del Cacho, I. Pérez-Álvarez, S. Zazo, E. Mendieta-Otero, H. SantanaSosa, J.M. Páez-Borrallo. Performance of an OFDM-CDMA HF Modem. Ninth International Conference on HF Radio Systems and Techniques. Bath, U.K. June 2003.

[15] NATO STANAG-4285. Characteristics of 1200/2400/3600 bits per second single tone modulators/demodulators for HF radio links. NATO Standardization Agreements. Edition 1, Amendment 1, 1990.

[16] Zazo, S.; F. Bader; J.M Paez Borrallo. Spread Spectrum OFDM Modem for HF Voice-Band Link Over Fading, European Transactions on Telecommunications, Volume 11, N6, pages: 593-598, November-December 2000. 